\title{
CONF-970503-269 \\ THE SLAC DAMPED DETUNED STRUCTURE: CONCEPT AND DESIGN
}

Norman M. Kroll,

Stanford Linear Accelerator Center, M/S 26, P.O Box 4349, Stanford, CA 94309

University of California, San Diego, La Jolla, CA 92093

\section{RECEIVED}

JUL 07997

The SLAC Pamped Detuned Structure (DDS) is an accelerator structure designed to suppress the long range transverse wakefields which limit the performance of high current multibunch accelerators. We discuss the conceptual considerations which have led to its development and discuss the steps involved in arriving at a design

Paper presented at the 1997 Particle Accelerator Conference

Vancouver, British Columbia, Canada

May 12-16, 1997

\section{DISCLAIMER}

\begin{abstract}
This report was prepared as an account of work sponsored by an agency of the United States Government. Neither the United States Government nor any agency thereof, nor any of their empleyees, makes any warranty, express or implied, or assumes any legal liability or responsibility for the accuracy, completeness, or usefulness of any information, apparatus, product, or process disclosed, or represents that its use would not infringe privately owned rights. Reference herein to any specific commercial product, process, or service by trade name, trademark, manufacturer, or otherwise does not necessarily constitute or imply its endorsement, recommendation, or favoring by the United States Government or any agency thereof. The views and opinions of authors expressed herein do not necessarily state or reflect those of the United States Government or any agency thereof.
\end{abstract}

${ }^{1}$ Supported by Department of Energy grant number DE-AC03-76SF00515 


\title{
THE SLAC DAMPED DETUNED STRUCTURE: CONCEPT AND DESIGN
}

\author{
Norman M. Kroll, \\ University of California, San Diego, La Jolla, CA 92093 \\ Stanford Linear Accelerator Center, M/S 26, P.O Box 4349, Stanford, CA 94309
}

\section{Abstract}

The SLAC Damped Detuned Structure (DDS) is an accelerator structure designed to suppress the long range transverse wakefields which limit the performance of high current multibunch accelerators. We discuss the conceptual considerations which have led to its development and discuss the steps involved in arriving at a design.

\section{INTRODUCTION}

The performance of long pulse train, high pulse current accelerators is limited by long range dipole wakefields. These wakefields distort particle orbits, thereby causing transverse emittance growth and eventually Beam Break Up. The SLAC Damped Detuned Accelerator Structure, the DDS, is being developed in order to mitigate this problem as it occurs for the design parameters of the linacs for the Next Linear Collider, parameters which include an X-band operating frequency with 120 nanocoulomb pulse trains of the order of fifty meters in length, but the concept is applicable to any long pulse train high pulse current accelerator.

\section{DESCRIPTION OF DDS 1}

The accelerator structures of the presently planned DDS series are composed of a sequence of 204 cells coupled by beam irises and terminated at each end with a matched coupler cell. The structure is driven at $11.424 \mathrm{GHz}$ through the upstream coupler, the downstream coupler is used to provide a matched termination. The cells are uniformly spaced at one third the $11.424 \mathrm{GHz}$ free space wavelength $(8.747 \mathrm{~mm})$ and must be dimensioned so as to maintain a uniform cell to cell phase advance of 120 degrees in the accelerating (monopole) mode. This requirement constitutes a constraint which must be respected when individual cell dimensions are varied so as to detune the dipole modes and the manifolds added so as to damp them.

The aim of Gaussian detuning [1] is to replace the narrow band of synchronous modes of each dipole band excited by an offset velocity of light bunch in a uniform structure with a broad band of discrete modes which by virtue of their amplitude and frequency distribution is Gaussian in a smoothed sense. If the frequency distribution were in fact a smooth Gaussian, then the wake function would exhibit a Gaussian decrease with time. The Gaussian is however truncated because the number of participating modes is finite, which imposes a finite frequency width on the Gaussian. Furthermore, since it is composed of a set of discrete modes, the mode amplitude density function is a sum of $\delta$ functions in frequency rather than a smooth function. On account of the truncation, the decrease is, on average, less than Gaussian after a time of the order of $\delta \mathrm{f} /\left(4 \pi \sigma_{\mathrm{f}}^{2}\right)$, where $\delta \mathrm{f}$ is the frequency width and $\sigma_{\mathrm{f}}$ the Gaussian width parameter. On account of the discreteness the destructive interference which caused the decrease becomes partially constructive at times of the order of the reciprocal mode separation, leading to a reappearance of the wake. These effects are exhibited in the detuned structure wake shown in Fig. 1.

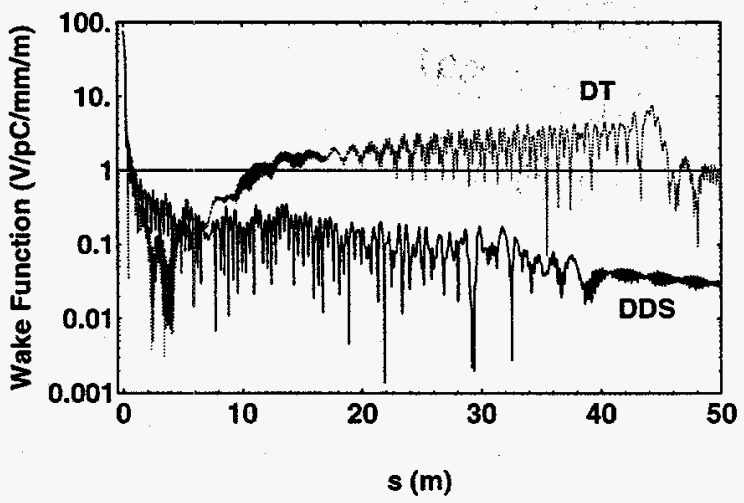

Fig 1: Wake function for DT and matched DDS 1

A careful balance between the Gaussian width $\sigma_{\mathrm{f}}$ and the frequency spread $\delta f$ is required to optimize the wake for the first few trailing bunches; the detuned structure of Fig. 1 has $\delta f$ equal to four times $\sigma_{r}$. With reference to trailing distance, the wake naturally separates into a short range (to be distinguished from the intra bunch wake) and a long range part.

In order to prevent the reappearance of the wake at larger time delays, damping is added to the detuned structure to form the DDS. The damping is provided by four waveguide-like structures equally spaced in azimuth around the accelerator structure and running its full length. In DDS 1, the first realization of the DDS concept, each waveguide is delineated by a rounded rectangular aperture in the disc containing the beam aperture which connects smoothly to a slot extension from the cylindrical wall of the cell. Because of their appearance and because they also serve as pumping manifolds, these structures are typically referred to as manifolds. Also because they are intended to drain dipole 
mode energy from the cells and carry it to loads at both ends of the manifolds, their function is manifold-like with respect to dipole mode energy as well.

In addition to the detuning related variation of cell dimensions, the manifold dimensions also vary along the structure to optimize cell to manifold coupling. Cross sections in the cell center and through the aperture disc as well as a perspective drawing are illustrated in [2], along with a cell in perspective (or cup) with the upper beam iris absent. The structure is fabricated by stacking such cups. The terminating loads involve a mitered 90 degree $\mathrm{H}$ plane bend, a 90 degree circular H-plane bend, a taper to standard waveguide, a window, and an external load. Two cells plus the coupler at each end of the structure are not connected to the manifolds to avoid conflict with the load assembly. The end section of the structure plus its load assembly is illustrated in Fig. 2.

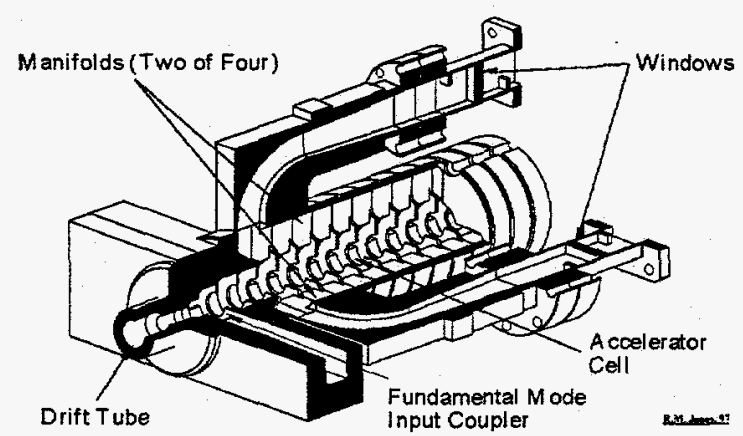

Fig 2: Cross-sectional view of DDS 1

\section{QUALITATIVE DESCRIPTION OF DDS DAMPING AND MANIFOLD RADIATION}

We begin by examining the properties of a strictly periodic structure formed by stacking a set of identical cells of typical cross section, say cell 106. Figure 3 shows a plot of the frequency of the lowest three modes as a function of phase advance per cell. The dots are points obtained by MAFIA simulation (see comparison to experiment in [3]), and the curves are obtained from an equivalent circuit theory $[4,5]$. The dashed curves correspond to equivalent circuit theory results obtained by setting the coupling of the cells to the manifold to zero. The large dashes correspond to the lowest two dipole modes of the manifold decoupled structure, the small dashes (fine-line in inset) to the manifold itself. The manifold has the character of a periodically shunted waveguide; only the lowest pass band is shown. One sees from Fig. 3 that the coupling has a significant effect only where the uncoupled curves approach one another, and that typical avoided crossing behavior is exhibited where the uncoupled curves cross (the "crossing point frequency" $f_{x}$ ). Examination of the electromagnetic field patterns shows a clear separation of modes into cell modes and manifold modes except at avoided crossing regions, where they mix. The synchronous mode (at the synchronous frequency $f_{s}$ where the light line and lower dipole curve cross) is virtually confined to the cell, therefore negligibly damped, and has a strong TM character, which is responsible for its strong coupling to a velocity of light beam.

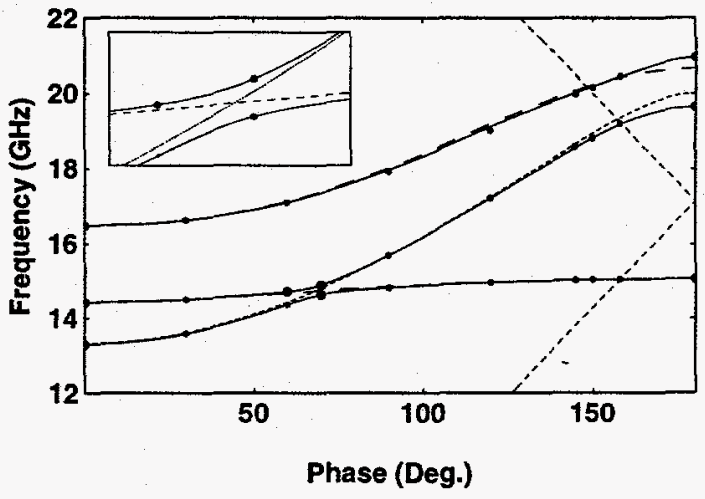

Figure 3: Brillouin Diagram for Cell 106 (Avoided Crossing shown inset)

Much of the behavior of a DDS can be understood by examining the aggregate of the individual cell properties described in the preceding paragraph. Figure 4 is a plot of the zero and $\pi$ phase advance frequencies, $f_{x}$, and $f_{s}$ all plotted as a function of cell number. The frequency range shown is restricted to the region relevant to the lower dipole mode. We do not discuss the upper dipole band because its contribution to the wake is negligible.

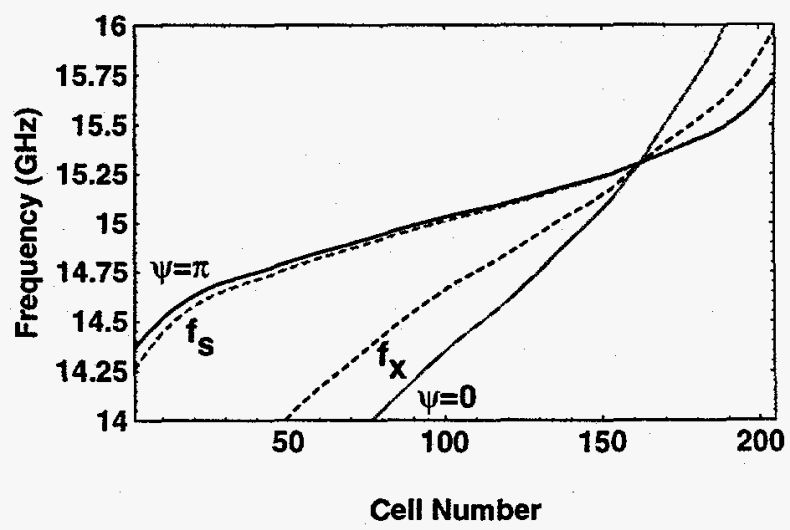

Figure 4: Mode limits, excitation point and damping point as a function of cell number

The curves are based on the uncoupled lower dipole curves (like that shown for cell 106 in Fig. 3) for an assortment of cells. The structure may be thought of as a propagating structure with slowly varying properties. There is a band of frequencies which appear on both phase advance curves. A mode within that band may be thought of as a wave which propagates back and forth between the turning points represented by these phase limits. Such modes are localized within the structure, 
being confined to the corresponding range of cells. At frequencies below this band modes are confined to a region between the upstream end of the structure and the cell on the zero phase advance line. Similarly modes at frequencies above this band lie between the cell on the zero phase advance line and the downstream end of the structure. The two phase advance curves cross because the cell to cell coupling changes from forward wave to backward wave for cells on the downstream side of the crossing region, and the modes with frequencies near this crossing are highly localized in the corresponding cells. An offset drive beam excites a mode at frequency $f$ at cells whose $f_{s}$ approximately coincides with $f$, and only the modes which have such cells contribute significantly to the wakefunction. Thus the frequency band of the wake function is limited to the band of synchronous frequencies. It is apparent from Fig. 4 that all such modes include cells with crossing frequency close to the mode frequency. Thus we have the following physical picture. A given mode is excited in the neighborhood of its synchronous cells. The excitation propagates back and forth between the zero and pi phase advance cells, passing the crossing point cells on each trip. The energy leaks into the manifold at the crossing point cells thus providing damping.

The confinement of most beam excited modes to the interior of the structure and the extreme localization of some are confirmed in equivalent circuit calculations and for azimuthally symmetric structures by full simulations [6] as well. The correlation of frequency with cell number is demonstrated in fig. 5 , and the relation between frequency and cell number which it implies is shown in fig. 6. Because this frequency at a given cell is shifted from the synchronous frequency of that cell, we refer to them as the coupled and uncoupled frequencies respectively. The short horizontal lines in fig 6 represent the number of cells in the localized offsets required to reach the level of the localization peaks shown in fig 5 and, are indicative of the sharpness of the localization. For application of the manifold power spectrum to monitoring beam position and structure alignment see [7, 8]. The following picture emerges of the damping process. To be specific we consider a particular mode, mode 100 at $14.836 \mathrm{GHz}$. The mode is localized between cells 48 and 148. The drive bunch launches a forward wave around cell 55 which propagates downstream. When it passes cells near 122 it loses power to the manifold, exciting in it a downstream wave. The remaining power is partially reflected at the coupling point and partially reflected at the downstream end of the mode. Only the latter portion contributes to a manifold wave in the upstream direction. In the perturbation theory limit the power coupled out is small and the forward and backward excitations of the manifold are equal. When the coupling to the manifold is large, however, one expects the downstream excitation of the manifold to predominate. Figure 5 indeed shows that the downstream preference occurs for virtually all the important modes, even those for which the relative position of the excitation and damping points are reversed. This reversal occurs only downstream from the point where the curves cross in Fig. 4, where the cell group velocity is negative. The beam induces a wave with forward phase velocity in the cells, but the excitation moves backwards and passes the coupling cell before it is reflected. Its phase velocity is still forward, however, so that it excites a forward wave in the manifold. Thus the expected preference is still downstream. This preference is in fact seen to be very large, one of many results in conflict with a perturbation treatment.

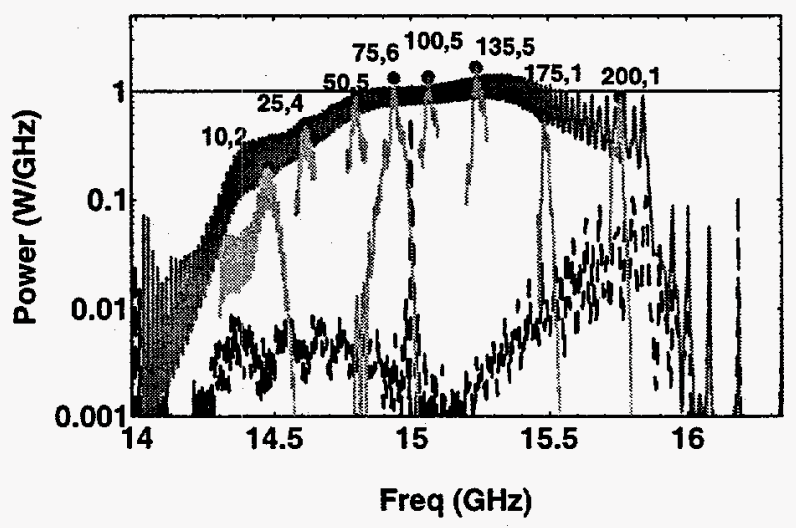

Fig 5: Power spectrum emitted from downstream DDS manifold with the power spectrum of localised offsets overlaid Upstream power spectrum shown dashed.

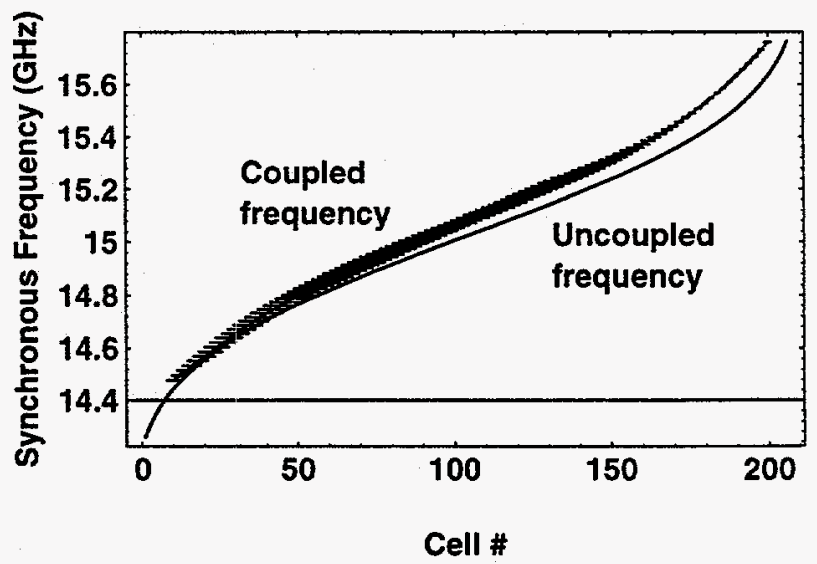

FIG 6: Coupled and uncoupled synchronous frequency vs cell number

\section{THE SPECTRAL FUNCTION}

The transverse momentum $p_{t}$ imparted to a charge $q_{w}$ witness bunch that has passed through an accelerator cavity of length $L$ trailing a charge $q_{d}$ drive bunch at distance $s$ with offset $r_{d}$, both traveling at velocity $c$, due to the dipole mode excitation left in the cavity by the drive 
bunch is expressed in terms of the wake function W(s) via the relation

$$
c p_{t}=q_{w} q_{d} r_{d} L W(s)
$$

$\mathrm{W}(\mathrm{s})$ is usefully expressed in terms of its spectral function $S(f)$ [5] as follows:

$$
W(s)=\theta(s) \int_{0}^{\infty} S(f) \sin (2 \pi s f / c) d f
$$

with

$$
S(f)=-4 \operatorname{Im}\{Z(f-j \varepsilon)\}
$$

where $Z(f)$ is the wake impedance, ie the Fourier transform of W(s) evaluated just below the real axis. The usually displayed wake envelope function $\hat{W}(s)$ is given by:

$$
\hat{W}(s)=\theta(s)\left|\int_{0}^{\infty} S(f) \exp (2 \pi j s f / c) d f\right|
$$

The definitions given here are appropriate to the wake function of a well aligned structure with a uniformly offset drive beam. Wake functions, wake moments, and their associated spectral function representations for more general situations are discussed in [9].

The computation of $S$ from the equivalent circuit representation of the structure is described in $[5,10]$. In the undamped case $S$ consists of a sum of $\delta$ functions generally written in the form:

$$
S(f)=2 \sum_{p} K_{p} \delta\left(f-f_{p}\right)
$$

where $K_{p}$ is the kick factor and $f_{n}$ the frequency of the modes [1]. In order to visualize its form for the undamped case it is useful to replace it by its smoothed version $S_{s}(f)$, obtained by replacing $\delta\left(f-f_{p}\right)$ by $1 /\left(f_{p+1}-f_{p}\right)$ over the interval $\left[f_{p}, f_{p+1}\right]$ and zero otherwise. The behavior of W(s) at the first trailing bunches is well represented by using $S_{s}$ in place of $S$. For DDS the spectral function is continuous in the pass bands spanned by the manifolds. The modes are damped in this frequency range, and (by design) the undamped modes outside this range have negligible kick factors. The appearance of $S$ is like a sum of lorentzians reflecting the underlying structure of damped modes and oscillating about the smoothed undamped $\mathrm{S}_{\mathrm{s}}$ (Fig. 7). If the structure is well damped, the widths are of the order of the mode separation so that the oscillations are quite gentle and for ideal damping would be barely discernible.
Apropos of the smoothed undamped spectral function $S$, we note that a good representation of the short range wake can also be obtained from an independent cell model, the "uncoupled model", as discussed in [1,11], and equally well from the associated smoothed spectral function, $S_{u}(f)$, a function which can be obtained without reference to an equivalent circuit. The frequencies and kick factors in the modal sum are simply the synchronous frequencies of the individual cells and their kick factors.

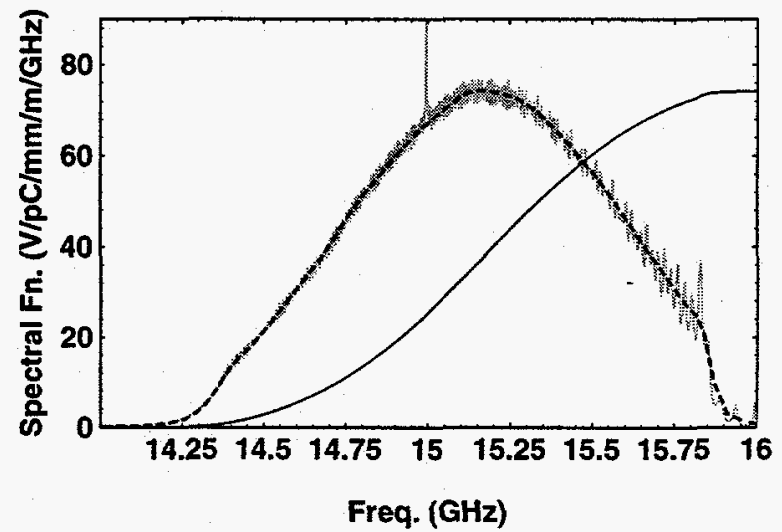

Fig 7: Matched DDS spectral function overlaying smoothed undamped spectral function

\section{DESIGN}

The design of a DDS begins with the selection of a basic cell-plus-manifold configuration and the dimensions to be varied to shape the detuning and damping profile. As discussed in $[12,13]$ the primary considerations are $Q$ (high) and shunt impedance (high) of the monopole mode, control of intra bunch transverse wakefields, and manufacturability. The manifolds are designed to be single mode in the lower dipole range and cutoff at the accelerator frequency. The pros and cons are discussed in [13], the main advantages, however, are compactness of design, simplification of fabrication, and of analysis. The basic design also includes the cell to manifold coupling configuration. The requirements are adequacy, adjustability, minimal degradation in cell $\mathrm{Q}$, shunt impedance, and pumping efficiency. A combination of openings in the cell walls, slots which intrude into the beam iris, and variation of manifold dimensions have been found to meet the requirements.

The next step in design is selection of a structure profile, ie cell parameters as a function of cell number. The procedure for the DDS is very similar to that of the detuned structure described in [11]. It is a two step process. Beginning with a qualitative idea of required detuning width, one designs a set $(\sim 11)$ of fiducial cells. The primary requirement is the tuning of the accelerator mode. This being done one needs the dispersion diagram for the lowest three dipole modes, the determination of the 
synchronous frequency $f_{s}$, and the kick factor for each of the fiducial cells. A smooth continuous mapping of cell dimensions and kick factors onto $f_{s}$ is then constructed. The selection of $S_{\mathrm{u}}(\mathrm{f})$ (typically a truncated Gaussian) provides as discussed in [13] an assignment of $f_{s}$ to each cell and allows one to compute and evaluate the short range wake in the uncoupled model. To proceed further it is necessary to use the dispersion diagrams to establish the circuit parameters of the equivalent circuit theory and to construct smooth continuous mappings of them on to $f_{s}$ as well. One can then construct $S_{s}$, the smoothed undamped but coupled spectral function and again compute and evaluate the short range wake. Should the two differ in detail in an unfavorable way, one could refine the $f_{s}$ distribution. This procedure was used to design DDS $3 \& 4$ from the fiducial cell set of DDS $2[12,14]$.

At this stage all the information is at hand to compute the complete damped wake function for matched loads from the full spectral function theory. Examples of the foregoing for matched manifold terminations are shown in Figs 1 and 7. Design of terminations is of course part of the design job. Up to now this has proved to be very important because even quite small reflections seriously degrade the wake. Reflection coefficients for DDS 1 are shown in [3], its highly oscillatory spectral function in Fig 8 , and its experimentally measured wake envelope function in Fig. 9.

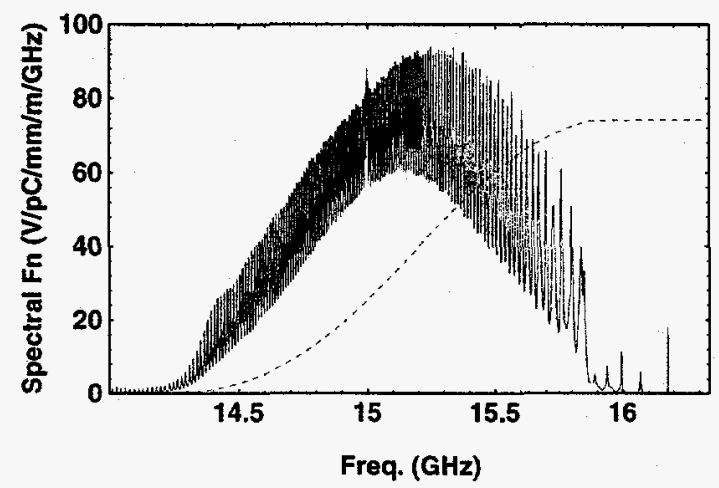

Fig 8: Spectral function for DDS 1 including VSWR of the manifold terminations.

Other examples, with reduced reflections and improved detuning configurations are shown in [12]. In order to optimize the damping profile it would be useful to have two or three sets of our $\sim 11$ fiducial cells. Then, using interpolation, one could choose manifold and cellmanifold coupling dimension profiles so as to lead to the smoothest spectral function. While at this stage matching seems to be more important than the damping profile, it is possible that the extreme sensitivity to reflection is related to the manifold being over coupled in the central region, and the contribution of the undercoupled cells at the doownstream end may not be as seriously affected by the reflections.

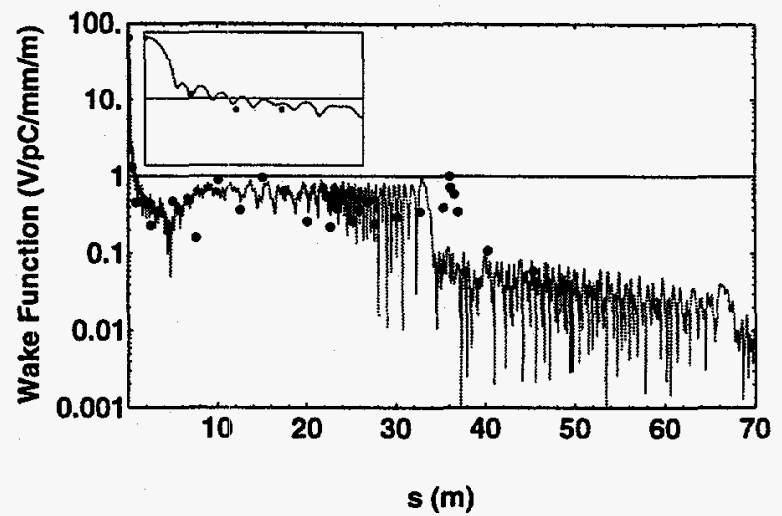

Fig 9: Calculated wake function for DDS 1. The dots indicate experimental data measured in the ASSET experiment. Shown inset is the short range wake function up to two metres.

\section{ACKNOWLEDGMENTS}

This work is supported by Department of Energy Grant DE-FG03-93ER40759 and Contract DE-AC0376SF00515. We have benefited greatly from the contributions of the DDS team. Special thanks are due R. H. Miller and R.M. Jones for their contributions to the material presented in this paper and for their assistance in its preparation.

\section{REFERENCES}

[1] K.L..F. Bane et al, Particle Accels., 42, p 123 (1993)

[2] K. Ko et al, Proc. PAC95, p 1058 (1995) SLAC-PUB 95-6884

[3] M. Seidel et al, Proc. Linac96, p 653 (1996) SLACPUB-7289

[4] RM. Jones et al, Proc EPAC 96, p 1292 (1996), SLAC-PUB-7187

[5] RM. Jones et al, Proc Linac96, p 650 (1996) SLACPUB-7287

[6] X-W Zhan \& K. Ko, paper 8p94, PAC'97

[7] M. Seidel, Paper 5B3, PAC97

[8] R.M. Jones et al, Paper 3w16, PAC97

[9] RM. Jones et al, Paper 3W15 PAC'97

[10] NM Kroll et al, Recent Results and Plans for the Future on SLAC DDS, Proc 7'th Workshop on Advance Accelerator Concepts, SLAC-PUB-7387 (1996)

[11] K.A. Thompson et al, Particle Accels., 47, p 65 (1994)

[12] R.M. Jones et al, Paper 3W14 PAC'97

[13] R.H.. Miller et al, Proc Linac96, p 644 (1996) SLAC-PUB-7288

[14] R.M. Jones et al, A Rapid Re-Design Method for the NLC DDS and Phase Stability of the Accelerating Mode: Implementation and Results, NLC-Note \#24 April 1997 


\section{DISCLAMMIER}

Portions of this document may be illegible in electronic image products. Images are produced from the best available original document. 
